\title{
Torcímetro digital para teste de torção em materiais e avaliação dos parâmetros elasto-plásticos de cisalhamento
}

\section{A digital torsimeter for torsion testing of materials and shear elasto-plastic parameters evaluation}

\author{
Maurício Ireno Betemps ${ }^{1}$, Renato Barbieri ${ }^{2}$, \\ Guilherme Ourique Verran ${ }^{2}$
}

\footnotetext{
${ }^{1}$ Programa de Pós-Graduação em Ciência e Engenharia de Materiais - PGCEM/UDESC CP: 89.219-710, Joinville, SC, Brasil.

${ }^{2}$ Departamento de Engenharia Mecânica - UDESC - Universidade do Estado de Santa Catarina. Campus Universitário 89219-710, Joinville, SC, Brasil.

e-mail: mibetemps@gmail.com, renato.barbieri@udesc.br, guilherme.verran@udesc.br
}

\begin{abstract}
RESUMO
Neste trabalho estão mostrados o projeto, os principais componentes e os resultados obtidos com o protótipo de um torcímetro digital que foi construído para medir a variação do ângulo de torção em ensaios mecânicos de materiais. Este dispositivo foi construído utilizando um encoder magnético, modelo AS5048A, com 14 bits de resolução e para o processamento dos dados foi utilizada uma placa microcontroladora Arduino Mega 2560. Além do baixo custo dos componentes eletrônicos e mecânicos, outra vantagem do sistema de medição desenvolvido é a grande quantidade de aplicativos gratuitos disponíveis para o processamento e aquisição dos dados com a placa microcontroladora utilizada. Foram realizados testes para verificar a exatidão nas leituras da variação angular e ensaios de torção com corpos de prova tubulares de alumínio (liga AA6351T6) para avaliar o desempenho do sistema de medição em ensaios monotônicos. A relação de Ramberg-Osgood foi utilizada para descrever o comportamento do material e as tensões de cisalhamento foram avaliadas empregando relações não lineares propostas por diferentes autores. Os dados obtidos indicam que o torcímetro digital apresentou resultados satisfatórios para a obtenção do ângulo de torção e as principais vantagens deste dispositivo incluem a medição do ângulo de torção sem contato, linearidade, boa acurácia, faixa de medição ilimitada, tamanho reduzido e baixo custo para confecção.
\end{abstract}

Palavras-chave: torcímetro, ensaio de torção, ângulo de torção.

\section{ABSTRACT}

In this work, the project, the main components and the results obtained with the prototype of a digital torsiometer that was built to measure the variation of the torsion angle in mechanical tests of materials are shown. A magnetic encoder model AS5048A was used in this device. This encoder has 14 bits of resolution and an Arduino Mega 2560 microcontroller board was used for data processing. In addition to the low cost of electronic and mechanical components, another advantage of the measurement system developed is the large amount of free applications available for processing and acquiring data with the microcontroller board used. Tests were carried out to verify the accuracy in the angular variation readings and torsion tests with aluminum tubular specimens (AA6351T6 alloy) to evaluate the performance of the measurement system in monotonic tests. The Ramberg-Osgood relationship was used to describe the behavior of the material and the shear stresses were evaluated using nonlinear relationships proposed by different authors. The results obtained indicate that the digital torsiometer presented satisfactory results for obtaining the torsion angle and the main advantages of this device include the measurement of the non-contact torsion angle, linearity, good accuracy over the entire measurement range, unlimited measurement range, reduced size and low cost for confection.

Keywords: Torsiometer, torsion test, twist angle. 


\section{INTRODUÇÃO}

Muitos componentes na indústria são submetidos a carregamentos cisalhantes ou combinações de carregamentos normais e cisalhantes. No entanto, devido à complexidade para realização de testes sob carga cisalhante e a menor disponibilidade de instalações para realização destes testes, as propriedades cisalhantes monotônicas e de fadiga estão disponíveis para um número reduzido de materiais [1]. Para suprir esta defasagem é fundamental o desenvolvimento de equipamentos e dispositivos pra realização de testes com cargas cisalhantes.

Os testes de torção normalmente são realizados utilizando corpos de prova tubulares ou sólidos. Os valores do torque e da variação do ângulo de torção são armazenados simultaneamente para o pósprocessamento das tensões, construção da curva tensão $\times$ deformação cisalhante e identificação dos parâmetros constitutivos do material. A Figura 1 ilustra um corpo de prova tubular típico e o comprimento Lg é a região indicada para as medições da variação do ângulo de torção.

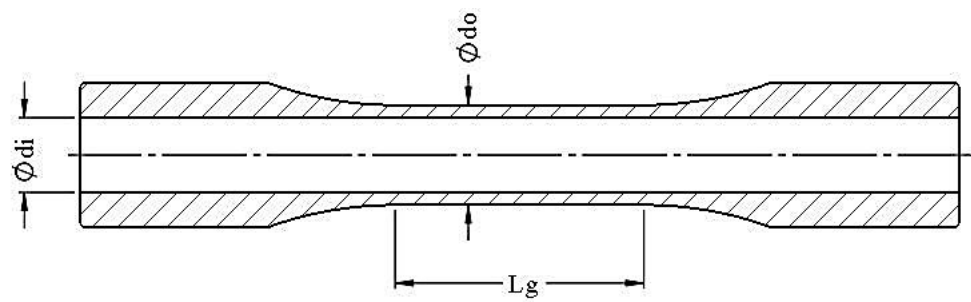

Figura 1: Corpo de prova típico para ensaio de torção.

Na região elástica da curva $\mathrm{T} \times \phi$ (torque $\times$ ângulo de torção) a tensão cisalhante, $\tau(\mathrm{d})$, varia linearmente ao longo da espessura do corpo de prova e para $\mathrm{d}_{\mathrm{i}} \leq \mathrm{d} \leq \mathrm{d}_{\mathrm{o}}$ o seu valor é dado por [2]:

$\tau(d)=\left[16 \mathrm{~T} / \pi\left(\mathrm{d}_{\mathrm{o}}^{4}-\mathrm{d}_{\mathrm{i}}^{4}\right)\right] \mathrm{d}$

onde $\mathrm{T}$ representa o torque externo, $\mathrm{d}_{\mathrm{o}}=2 \mathrm{r}_{\mathrm{o}}$ é o diâmetro externo do tubo e $\mathrm{d}_{\mathrm{i}}=2 \mathrm{r}_{\mathrm{i}}$ é o seu diâmetro interno.

Entretanto, na região elasto-plástica o comportamento da tensão cisalhante passa a ser não linear na parede do corpo de prova e o seu cálculo é um pouco mais complexo. A norma ASTM E2207-15 [2] sugere adotar a hipótese de que a tensão cisalhante é distribuída uniformemente ao longo da parede dos corpos de prova com paredes finas e que é aceitável estimar o valor médio da tensão com a seguinte expressão [3]:

$\tau(d)=16 T / \pi\left[\left(d_{0}^{2}-d_{i}^{2}\right)\left(d_{o}+d_{i}\right)\right]$

De acordo com WU et al. [4] o valor da tensão cisalhante na superfície externa dos corpos de prova tubulares com carregamentos no regime elasto-plástico é dado por:

$\tau_{\mathrm{o}}=\left\{\tau_{\mathrm{i}} \mathrm{r}_{\mathrm{i}}^{3}\left[1-(1+2 \mathrm{c}) \mathrm{D}\left(\mathrm{H}_{\varepsilon}\right)+\mathrm{cD}\left(\mathrm{A}_{\varepsilon}\right)\right]+\mathrm{D}(\mathrm{T}) / 2 \pi\right\} / \mathrm{r}_{\mathrm{o}}^{3}\left[1-\mathrm{D}\left(\mathrm{H}_{\varepsilon}\right)\right]$

sendo que $\tau_{\mathrm{o}}$ e $\tau_{\mathrm{i}}$ representam a tensão cisalhante no raio externo e no raio interno, respectivamente. $\mathrm{D}($.) é o operador diferencial $\mathrm{D}()=.3()+.\theta \mathrm{d}(.) / \mathrm{d} \theta$ e a variação do ângulo de torção por unidade de comprimento é representada por $\theta$. O parâmetro $c$ é um escalar utilizado para especificar a espessura t da parede do tubo em função do raio interno, $\mathrm{t}=c \mathrm{r}_{\mathrm{i}}$. $\mathrm{O}$ torque, a deformação axial e a deformação no diâmetro são identificados por $\mathrm{T}, \mathrm{A}_{\varepsilon}$ e $\mathrm{H}_{\varepsilon}$, respectivamente.

Quando os testes de torção são realizados com deslocamento axial restrito nas extremidades dos corpos de prova as deformações $\mathrm{A}_{\varepsilon}$ e $\mathrm{H}_{\varepsilon}$ são nulas e a equação (3) reduz-se à expressão de BROWN [5] que também é sugerida na norma ASTM E2207-15 [2] para os corpos de prova com parede espessa:

$\tau_{\mathrm{o}}=\tau_{\mathrm{i}}\left(\mathrm{r}_{\mathrm{i}} / \mathrm{r}_{\mathrm{o}}\right)^{3}+(3 \mathrm{~T}+\theta \mathrm{dT} / \mathrm{d} \theta) / 2 \pi \mathrm{r}_{\mathrm{o}}{ }^{3}$

O valor da tensão cisalhante $\tau_{\mathrm{o}}$ das equações (3) ou (4) é avaliado a partir da curva experimental $\mathrm{T} \times \phi$, sendo necessário um procedimento incremental de cálculo porque a tensão cisalhante possui comportamento não linear ao longo da parede do corpo de prova e o valor de $\tau_{\mathrm{i}}$ também é desconhecido. BROWN [5] e WU et al. [6] descrevem procedimentos de cálculo incremental para avaliar estas tensões.

Os corpos de prova sólidos com restrição de movimento axial nas duas extremidades possuem $\mathrm{r}_{\mathrm{i}}=\mathrm{A}_{\varepsilon}=\mathrm{H}_{\varepsilon}=0$. Com estas simplificações a equação (3) reduz-se à conhecida equação de NADAI [7]: 
$\tau_{0}=(3 \mathrm{~T}+\theta \mathrm{dT} / \mathrm{d} \theta) / 2 \pi \mathrm{r}_{0}{ }^{3}$

Assumindo a hipótese de isotropia plana, a deformação de cisalhamento $\gamma(\mathrm{r})$ pode ser calculada usando a variação do ângulo de torção através da seguinte relação [5]:

$\gamma(\mathrm{r})=\mathrm{r} \Delta \phi / \Delta \mathrm{L}=\mathrm{r} \theta$

onde $\Delta \phi$ indica a variação do ângulo de torção medido entre dois pontos separados por $\Delta \mathrm{L}$ no comprimento útil do medidor. Esta expressão é válida para os regimes elástico e elasto-plástico.

Observando as expressões analíticas para avaliação das tensões e deformações, equações (3) até (6), verifica-se que para assegurar bons resultados para as tensões, a partir dos dados do ensaio de torção, é necessário medir a variação do ângulo de torção $\Delta \phi$ e o torque com boa acurácia. Além disso, é necessário empregar uma técnica numérica adequada para obter também bons resultados para a derivada do torque, $\mathrm{dT} / \mathrm{d} \theta$.

Um dispositivo para medição do ângulo de torção sobre o comprimento útil dos corpos de prova já aparece na patente americana de um torcímetro registrada em 1928 por John Hammond Smith [8] que utiliza um 'transferidor' como instrumento auxiliar para a medição do ângulo de torção. Este mesmo princípio utilizado por Smith [8] em 1928 ainda é utilizado atualmente, como mostra o torcímetro ilustrado na Figura 2a. O instrumento ilustrado tem resolução de $0,1^{\circ}$ e não apresenta limitação na sua faixa de medição [9]. $O$ custo deste tipo de acessório é relativamente baixo, porém, como apresenta o sistema de medição baseado na utilização de Escala Fixa e Nônio não é possível utiliza-lo em aplicações que exijam múltiplas medições devido à inviabilidade para o registro destes dados (como exemplo, a obtenção da curva torque - ângulo ou ensaios cíclicos em torção).

Para a medição do ângulo de torção $(\Delta \phi)$ nos ensaios de torção geralmente são utilizados transdutores de deslocamento linear, potenciômetros, resolver, encoder e extensômetros para a medição indireta do ângulo de rotação entre duas posições no corpo de prova. Dentre estes é mais comum encontrar torcímetros construídos utilizando os transdutores lineares com baixo custo (relógio comparador ou LVDT), porém o uso destes transdutores é restrito à própria faixa de medição e à perda de contato entre a base de apoio, Figura $2 \mathrm{~b}$. Além disso, a relação entre o ângulo de giro e o deslocamento do transdutor é não linear em toda a faixa de medição [10]. Um exemplo deste tipo de torcímetro é mostrado na Figura 2c.

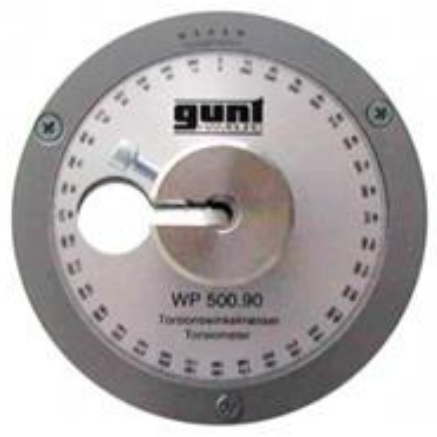

a) Torcimetro comercial com escala fixa e Nônio [9].

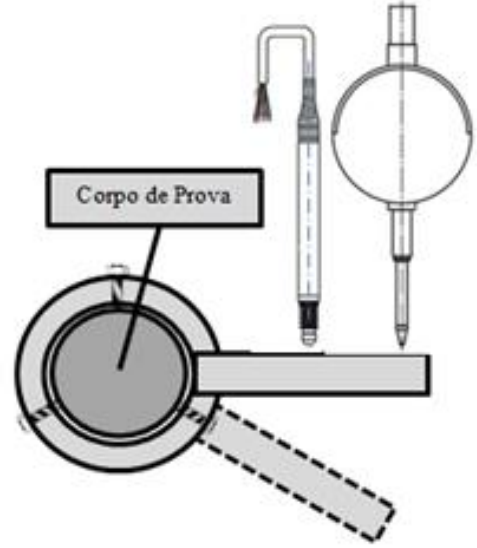

b) Esquema de medição empregando relógio comparador éou LVDT.

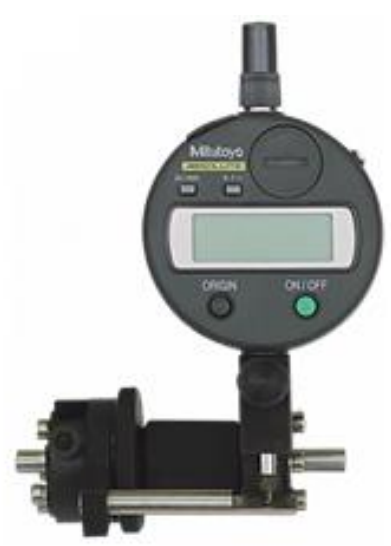

c) Transdutor comercial com Relógio Comparador [11]

Figura 2: Medição do ângulo de torção com Nônio e com Transdutores de Deslocamento Linear.

No torcímetro desenvolvido por SWIFT [12] foi utilizado um potenciômetro para as medições indiretas da variação do ângulo de torção. Os potenciômetros possuem custo relativamente baixo, porém não são muito precisos nas medições de ângulo pequenos. Estes elementos apresentam uma amplitude limitada de rotação e por possuírem contato entre uma pista e um cursor pode ocorrer desgaste nessas peças em determinadas aplicações, como por exemplo, ensaios cíclicos. De acordo com a forma construtiva, estes elementos podem apresentar relação linear ou não linear entre os sinais de entrada e saída.

SANTOS e BARBIERI [10] e SCHERER et al. [13] utilizaram a extensometria para a medição do deslocamento angular sobre o comprimento útil de corpos de prova cilíndricos. Nos dois casos foram colados 
extensômetros sobre uma viga (elástica) engastada em uma de suas extremidades e apoiada na outra extremidade em um disco excêntrico. Embora não possua limitação do ângulo máximo de torção, a relação entre a entrada e saída é aproximadamente linear em pequenas faixas de medição. Os dispositivos utilizados por SANTOS e BARBIERI [10] e SCHERER et al. [13] estão ilustrados nas Figuras 3a e 3b respectivamente.

PINTÃO et al. [14] desenvolveram um dispositivo para medição do módulo de torção (G) em amostras de polímeros, onde o ângulo de torção foi medido através de um sensor de movimento rotacional (RMS) com $0,25^{\circ}$ de resolução. O deslocamento angular ocorrido nas amostras é transmitido para o sensor RMS em um eixo paralelo ao do corpo de prova através de um sistema formado por braços de alavanca, cabos e polias. Neste sistema o ângulo de torção é medido em um componente da máquina, fixado em uma das extremidades do corpo de prova.

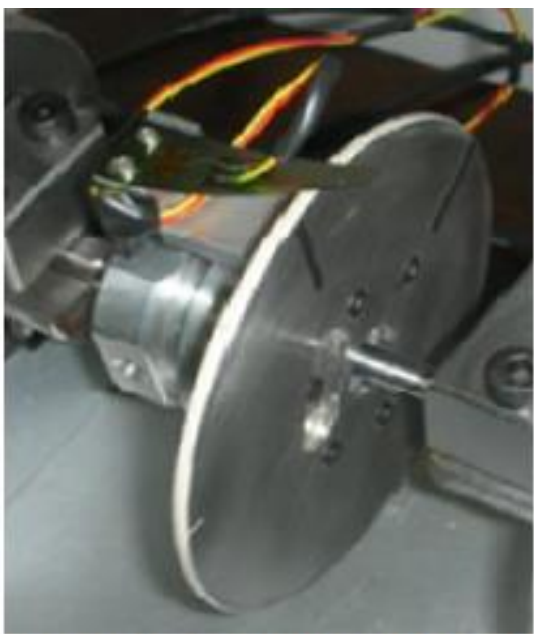

a) Dispositivo utilizado por SANTOS e BARBIERI [10]

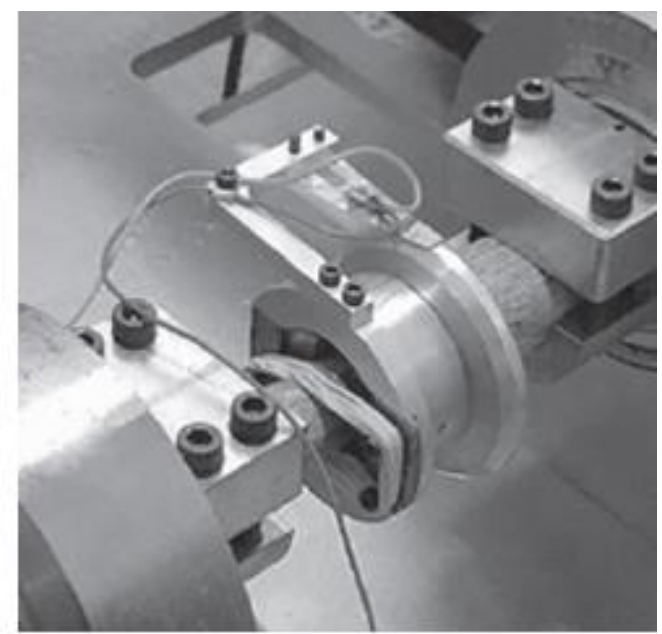

b) Dispositivo utilizado por SCHERER est al [13]

Figura 3: Torcímetro com princípio de medição baseado em extensometria.

Com o objetivo de superar as limitações inerentes a utilização de sensores com contato, tem-se buscado opções de sensores sem contato para medições de deslocamento linear e angular. No trabalho realizado por KAKALEY et al. [15] foram utilizados sensores de relutância variável (VR) sem contato em um sistema para medição simultânea do torque, velocidade e deslocamento axial em eixos de alta velocidade. Este sistema consiste em seis sensores VR montados em uma carcaça que envolve duas rodas dentadas e o eixo rotativo.

LIU et al. [16] desenvolveram um dispositivo para medição do ângulo de torção em fios de arame, neste estudo foi utilizado um sensor de deslocamento a laser para determinar a posição angular de uma haste fina fixada ao corpo de prova. YU et al. [17] também desenvolveram um dispositivo para medição do ângulo de torção em fios de arame, neste trabalho foi utilizada uma câmera digital para realização das medições através do processamento das imagens obtidas da posição angular de um anel circular conectado a amostra.

TORO et al. [18] analisaram a resposta elasto-plástica de corpos de prova cilíndricos submetidos a ensaios de torção. A medição do ângulo de torção foi realizada através da correlação de imagem digital 2D, onde, não havendo variação do raio durante o ensaio de torção, a medição do ângulo de torção pode ser obtida através da projeção sobre um plano das sucessivas posições de um ponto sobre a superfície do corpo de prova. Este sistema permitiu a medição de ângulos de até aproximadamente $70^{\circ}$, correspondente à faixa em que é possível a captura da imagem pela câmera. Conforme apresentado por Traphöner et al. [19], testes de torção em materiais metálicos dúcteis apresentam deformações reais muito superiores a 1 , sendo a medição destas altas deformações impraticáveis através do método da correlação de imagem digital convencional (DIC), desta forma, as curvas tensão-deformação cisalhantes ficam limitadas e as deformações de fratura para materiais dúcteis não podem ser determinadas. Esta condição exige que os testes sejam realizados em múltiplas etapas, onde sempre um novo padrão óptico é aplicado à amostra.

Os torcímetros construídos utilizando encoders e resolvers apresentam boa resolução, são robustos, de montagem relativamente fácil, porém alguns modelos possuem custo relativamente alto que podem restringir o seu uso [20].

O torcímetro digital descrito neste trabalho foi construído com o objetivo de atender aos requisitos de precisão e exatidão na leitura do ângulo de torção; com baixo custo de fabricação e facilidade para o processamento dos dados do ensaio. Na construção deste dispositivo se teve também como propósito a 
definição de um sistema de fácil montagem, ampla faixa de medição, saída digital com taxa de transferência de dados adequada para ensaios monotônicos e cíclicos, assim como, a eliminação dos erros decorrentes das folgas e deformações dos componentes encontrados em sistemas de medição com contato e sistemas de fixação das amostras. A descrição das principais características do dispositivo e de seus principais componentes é realizada no início deste trabalho. A seguir, são apresentados testes executados para verificar a acurácia das medições do ângulo de torção e no final do trabalho são mostrados os resultados da torção de um corpo de prova tubular fabricado com a liga de alumínio AA6351T6. Nesta última etapa são detalhados os procedimentos numéricos utilizados no tratamento dos dados experimentais para obter as tensões cisalhantes e determinar os parâmetros constitutivos da relação de Ramberg-Osgood, utilizada para modelar a resposta do material submetido ao ensaio de torção.

\section{MATERIAIS E MÉTODOS}

\subsection{O torcímetro digital}

O torcímetro deste trabalho foi construído utilizando um sensor magnético (AS5048A) para a medição sem contato do ângulo de torção e devido a esta característica praticamente não existe limite máximo para a faixa de medição. Na Figura 4 é mostrado o torcímetro acoplado a um corpo de prova tubular e a uma placa microcontroladora Arduino Mega 2560 que é utilizada para aquisição dos dados da leitura digital do sensor magnético.

O AS5048A é um encoder magnético com 14 bits de resolução e saída digital. Para a medição do ângulo de torção sem contato é utilizado um ímã de referência que deve ser posicionado à distância de $0,5 \mathrm{a}$ 2,5 mm do encoder, Figura 5. É um sensor que pode ser utilizado para medir ângulos sem restrições e após linearização e média com auxílio do microcontrolador é esperada precisão da ordem de $0,05^{\circ}$ para os valores medidos [21]. A placa do sensor mede $28 \times 22 \mathrm{~mm}$ e o ímã possui $\varnothing=6 \mathrm{~mm}$ e altura $\mathrm{h}=2.5 \mathrm{~mm}$. No projeto do dispositivo foi previsto uma distância de $2 \mathrm{~mm}$ entre o imã e o encoder. Para posicionamento radial do imã existe um rebaixo na ponta do eixo onde o mesmo é fixado com cola. A placa com o sensor AS5048A pode ser encontrada ao custo aproximado de US\$25,00. A alimentação da placa AS5048A é realiza através de uma fonte de corrente contínua de $5 \mathrm{~V}$.

A placa com o microprocessador Arduino Mega 2560 é utilizada para a leitura dos dados digitais. Dois fatores foram relevantes para a seleção deste microprocessador: o custo aproximado de US $\$ 25,00$ e a quantidade de aplicativos gratuitos disponíveis na internet para o gerenciamento dos dados de entrada e saída do AS5048A com esta placa.

Os componentes (1), (2) e (3) mostrados na Figura 5 foram fabricados em alumínio e o movimento relativo de rotação sem restrição entre (1) e (2) é garantido pelo rolamento inserido entre estas peças. Nestas duas peças são fixadas com parafusos de aço inoxidável duas placas com formato em $\mathrm{V}$ para o apoio do corpo de prova, Figura 6. As duas placas foram fabricadas em alumínio e possuem espessura de $1 \mathrm{~mm}$. A distância entre estas duas placas é de $25 \mathrm{~mm}$ e corresponde ao comprimento $\Delta \mathrm{L}$ que aparece nas fórmulas para cálculo do ângulo de torção por unidade de comprimento.

Durante o teste de torção, o movimento relativo entre as peças (1) e (2) é transmitido pela engrenagem de dentes retos com 66 dentes para o pinhão com 18 dentes, o qual, está acoplado ao eixo de alumínio onde é fixado o ímã. Então, o valor da variação angular entre os apoios do corpo de prova é dado pelo valor lido no encoder magnético multiplicado por 18/66 (razão de transmissão de movimento). 


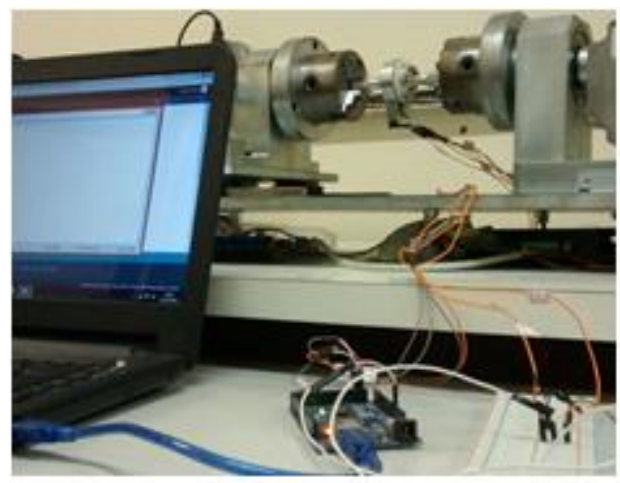

a) Cadeia de medição

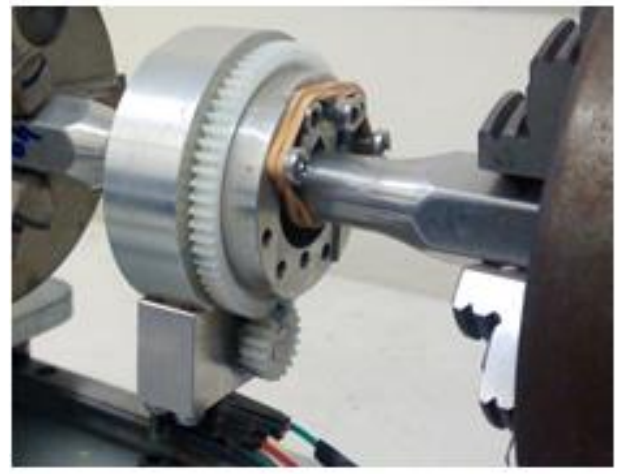

b) Detalhe da fixação do torcimetro no corpo de prova

Figura 4: Montagem do sistema de medição.

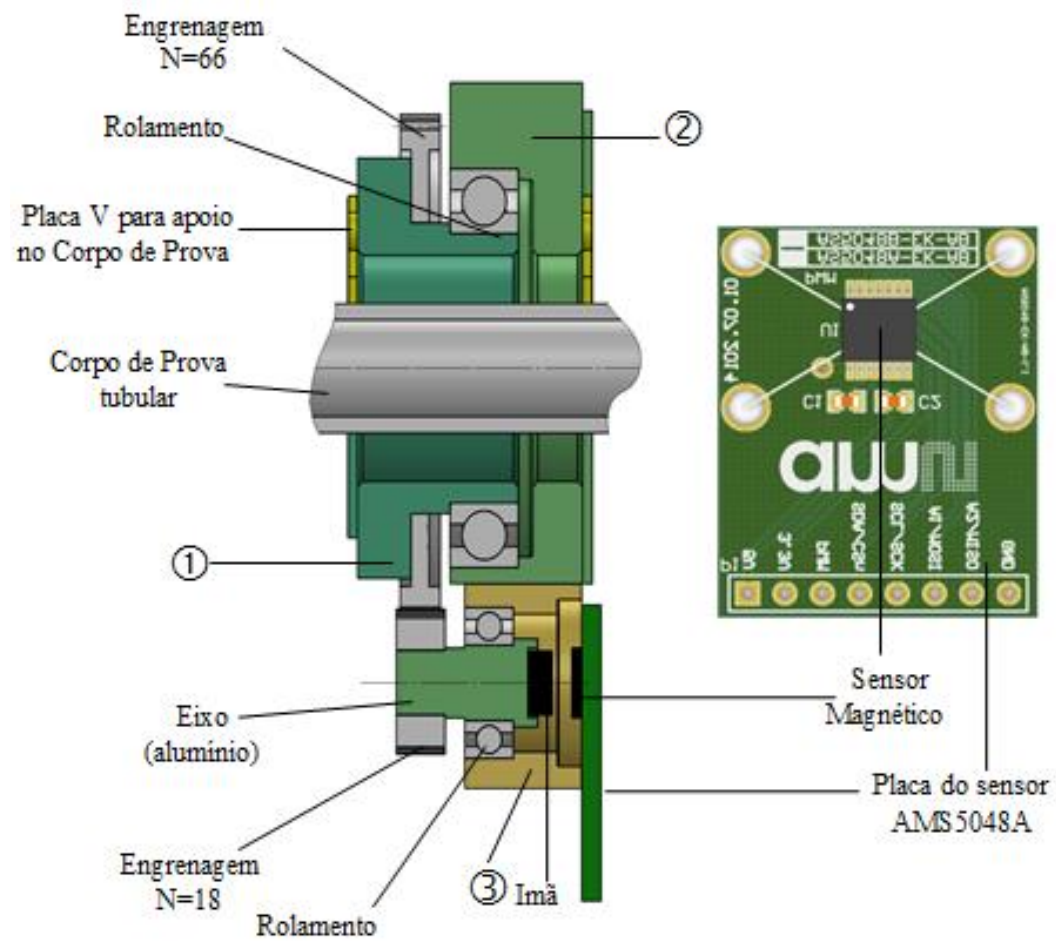

Figura 5: Principais componentes do torcímetro digital

O elemento elástico visto na Figura 6 é utilizado para pressionar o corpo de prova contra os apoios em V com o objetivo de evitar o seu deslizamento angular. A aplicação dos elementos elásticos para fixação também tem por objetivo evitar danos ao dispositivo em eventuais deslocamentos excessivos ocorridos no 
momento da ruptura.

Foi possível obter a taxa de 40 leituras por segundo quando foi realizada apenas a leitura dos dados do encoder. Para a leitura simultânea dos dados do encoder e de uma célula de carga obteve-se a taxa de 17 leituras por segundo.

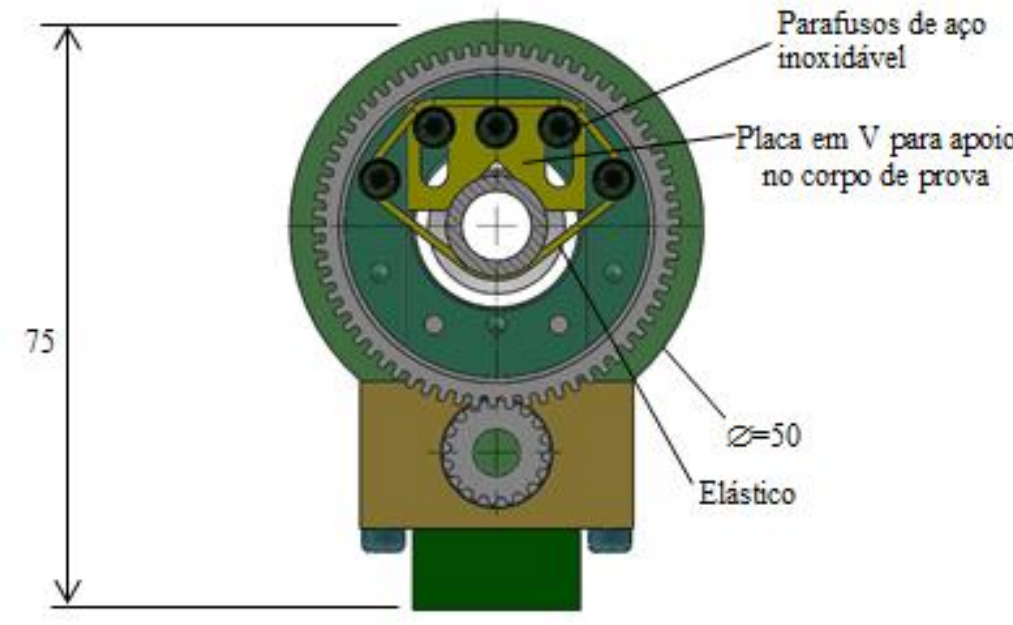

Figura 6: Dimensões principais e sistema de fixação do torcímetro no corpo de prova.

\subsection{Testes preliminares}

Testes preliminares foram realizados para verificar a acurácia do ângulo lido com este dispositivo digital. O motor de passo, utilizado no acionamento da máquina de torção mostrada na Figura 7a permite controle angular com resolução de $0,03^{\circ}$ e, por este motivo as comparações foram realizadas tomando os valores desta máquina como referência.

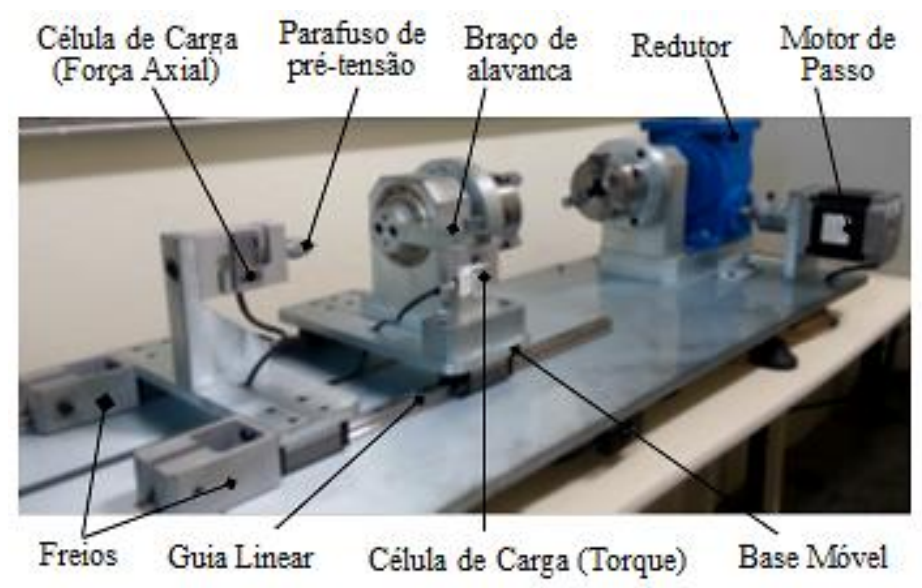

a) Máquina para ensaio de torção

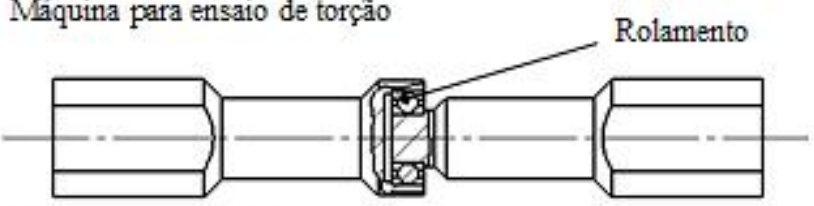

b) Dispositivo de teste

Figura 7: Equipamento de referência para comparação do ângulo medido.

O torcímetro digital foi acoplado ao dispositivo ilustrado na Figura 7b. Como este dispositivo permite rotação livre entre as suas extremidades, o valor registrado pelo torcímetro deveria ser igual ao valor do ângulo de referência. A diferença $\Delta \Phi$ encontrada entre estes dois valores é mostrada na Figura 8.

Os dados desta figura mostram $-0.194^{\circ} \leq \Delta \Phi \leq 0.151^{\circ}$ com valor médio igual a $0.0114^{\circ}$ e desvio padrão 
de $0.0846^{\circ}$. Devido à transmissão de movimento com o par de engrenagens o ângulo medido pelo encoder digital é 66/18 vezes o ângulo de referência. Então, uma volta no eixo de referência corresponde a 3,666 voltas do eixo do encoder e isto pode explicar o padrão aproximadamente periódico que aparece na Figura 8.

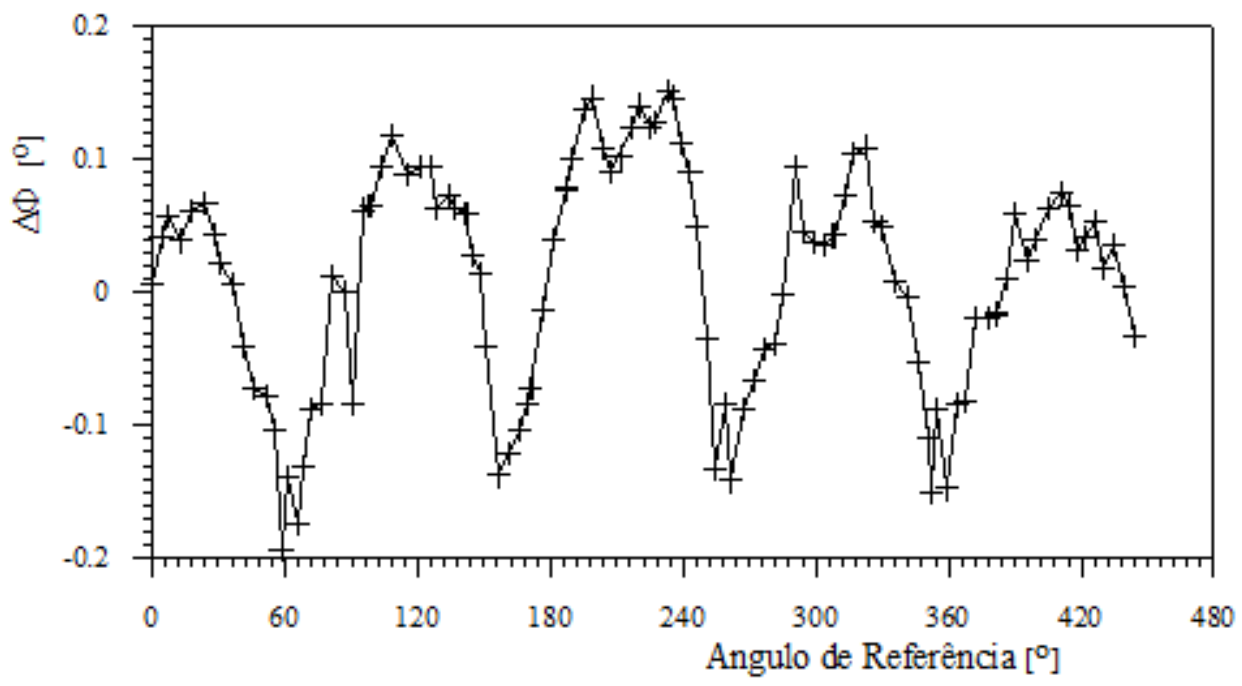

Figura 8: Diferença entre o ângulo do torcímetro e o ângulo de referência.

\section{RESULTADOS}

\subsection{Teste de torção}

As figuras 9a e 9b ilustram as curvas torque $\times$ ângulo de torção para a liga forjada de alumínio AA6351T6 obtidas no ensaio de corpo de prova tubular com dimensões $d_{0}=11,96 \mathrm{~mm}$, di=9,10 $\mathrm{mm} \mathrm{e} \mathrm{L}_{\mathrm{g}}=25 \mathrm{~mm}$.

O módulo de cisalhamento $(\mathrm{G})$ pode ser estimado utilizando a inclinação da curva experimental $\mathrm{T} \times \phi$ na região elástica (ou quase elástica) que é a região próxima da origem, Figura 9b. Nesta região,

$\mathrm{T} \cong \phi \mathrm{GJ} / \Delta \mathrm{L}=1357,41 \phi$

sendo $\mathrm{J}=\pi\left(\mathrm{d}_{\mathrm{o}}{ }^{4}-\mathrm{d}_{\mathrm{i}}{ }^{4}\right) / 32 \cong 1335,52 \mathrm{~mm}^{4}$ e $\Delta \mathrm{L}=25 \mathrm{~mm}$. Então, $\mathrm{G} \cong 25409,76 \mathrm{~N} / \mathrm{mm}^{2}$.

Para o processamento dos valores da tensão cisalhante na região não linear é mais conveniente construir a curva do ângulo de torção em função do torque e obter uma curva aproximada para $\phi(T)$ utilizando o Método dos Mínimos Quadrados (MMQ). Esta curva é mostrada na Figura 10 e para dT/d $\phi \geq 0$ o seu formato é semelhante às curvas de Ramberg-Osgood e a expressão ajustada para $\phi(\mathrm{T})$ é dada por:

$\phi \cong(\mathrm{T} / 1367,11)+(\mathrm{T} / 35,60)^{7,245} \quad \mathrm{~T} \leq 42,2 \mathrm{Nm}$

A relação de Ramberg-Osgood (RO) foi utilizada nesta aplicação para descrever o comportamento da curva $\tau \times \gamma$. Nesta relação a deformação cisalhante é obtida com a soma da parcela elástica e da parcela plástica e obtida por:

$\gamma=\gamma_{E}+\gamma_{P L}=(\tau / G)+(\tau / H)^{1 / n}$

sendo que $\mathrm{G}$ representa o módulo de cisalhamento, $\mathrm{H}$ é o coeficiente de resistência e $\mathrm{n}$ é expoente de encruamento.

Esta equação possui três parâmetros desconhecidos: G, H e n. Entretanto, o valor de G é obtido com o ajuste linear dos dados ao redor da origem da curva $\gamma \times \tau$, pois $\mathrm{d} \gamma / \mathrm{d} \tau=1 / \mathrm{G}$ em $(\gamma, \tau)=(0,0)$. Após este ajuste preliminar para estimar o valor de $\mathrm{G}$ os outros dois parâmetros podem ser identificados com auxílio da equação (2) ou (4). 


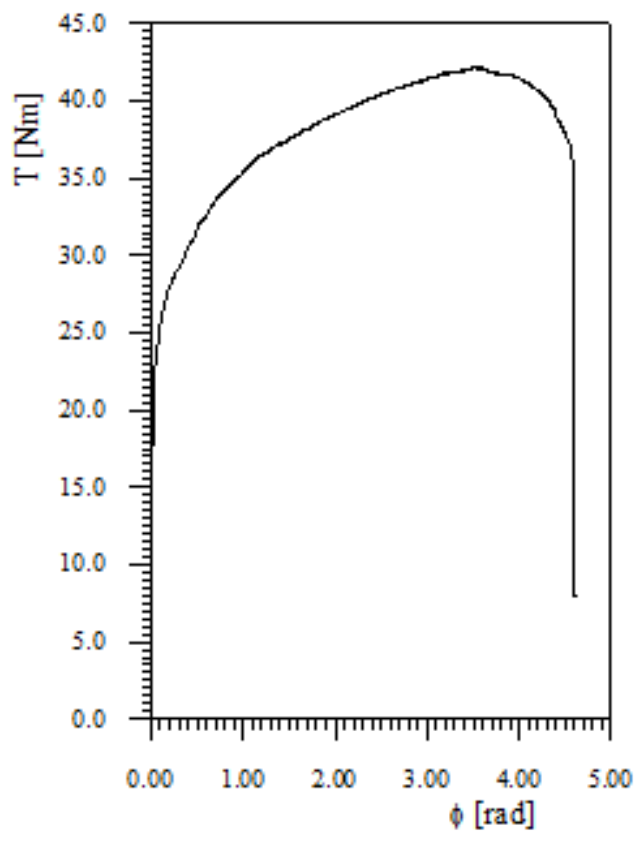

a) Curva Experimental $\mathrm{T} \times \phi$

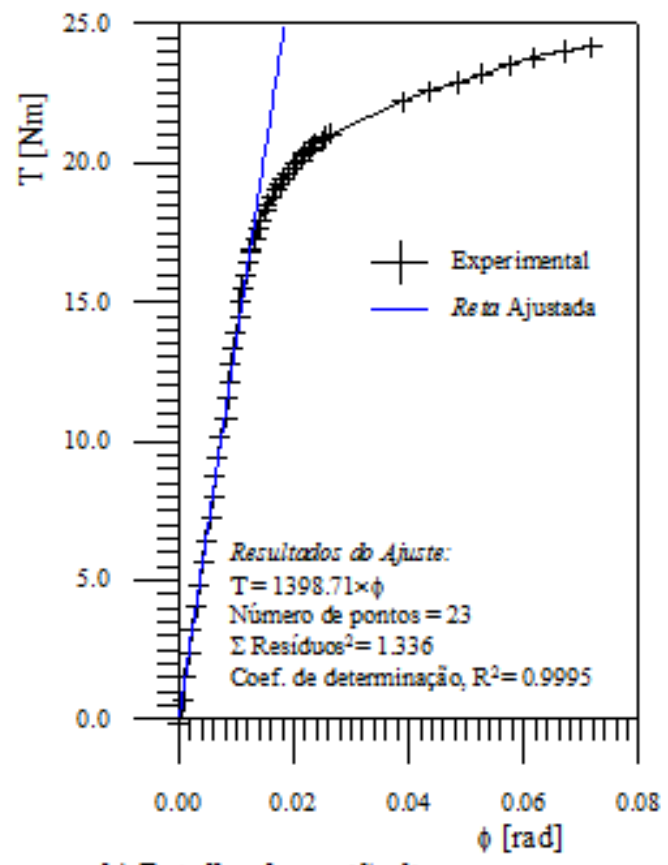

b) Detalhe da região linear

Figura 9: Dados experimentais.

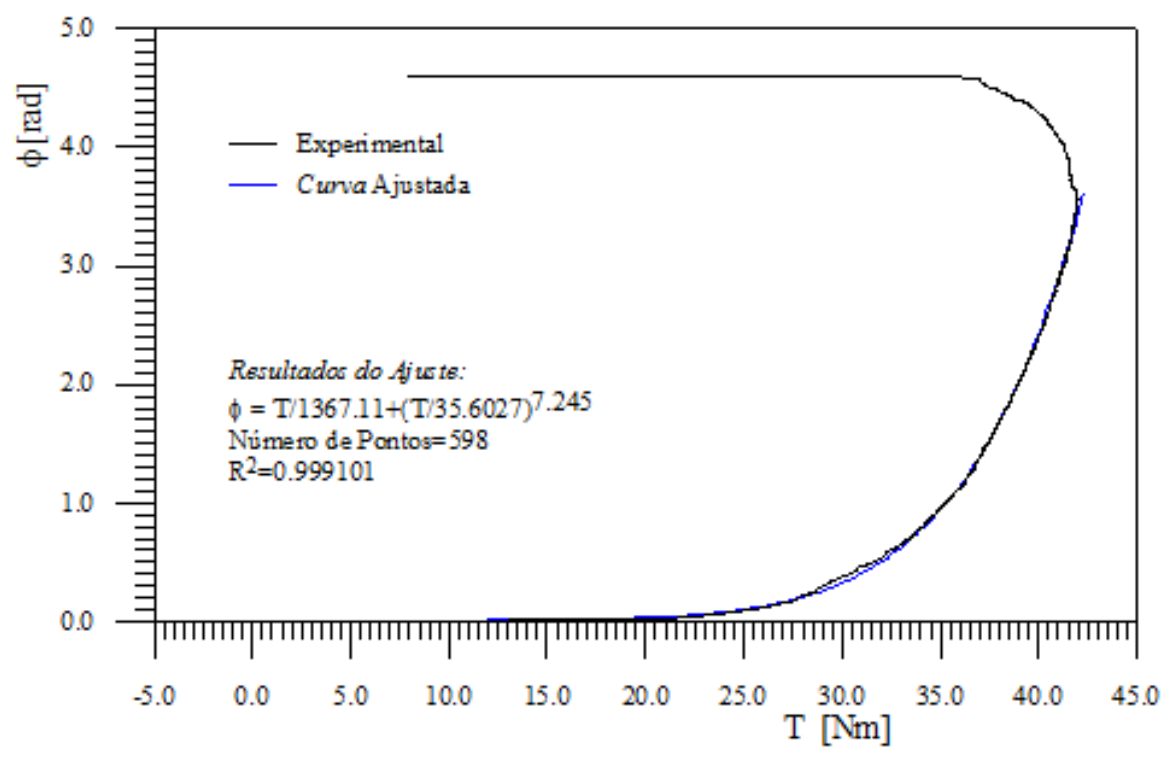

Figura 10: Dados experimentais e curva ajustada para $\phi(T)$.

\subsection{Identificação dos Parâmetros do Material a partir da equação (2) - Parede Fina.}

O tratamento dos dados experimentais com a equação (2) é mais conveniente, pois a relação entre a tensão cisalhante e o torque permanece linear mesmo no regime não linear de carga. Isolando $\gamma$ na equação (6), T na equação (2) e substituindo na equação (8) resulta:

$\gamma=\phi\left(\mathrm{r}_{\mathrm{o}} / \mathrm{L}\right) \cong\left(\mathrm{r}_{\mathrm{o}} / \mathrm{L}\right) \cdot\left[\left(\mathrm{c}_{1} \tau / 1367,11\right)+\left(\mathrm{c}_{1} \tau / 35,60\right)^{7,245}\right]$

$\operatorname{com} c_{1}=(\pi / 16) \cdot\left(d_{o}^{2}-d_{i}^{2}\right) \cdot\left(d_{o}+d_{i}\right)$.

$\gamma \cong\left[\left(\tau / 22947,19 \times 10^{6}\right)+\left(\tau / 174,13 \times 10^{6}\right)^{7,245}\right]$

Comparando a equação (11) com a relação de Ramberg-Osgood identificam-se os parâmetros 
monotônicos do material, G=22947,3 MPa, H=174,2 Mpa e nミ0,138. De imediato é notado que o valor obtido para $\mathrm{G}$ com esta abordagem possui diferença acentuada com relação ao valor $\mathrm{G} \cong 25409,8 \mathrm{~N} / \mathrm{mm}^{2}$ obtido a partir da inclinação inicial da curva experimental $\mathrm{T} \times \phi$, Figura $9 \mathrm{~b}$.

\subsection{Identificação dos Parâmetros do Material utilizando a equação (4) - Parede Espessa.}

O tratamento dos dados utilizando a equação (3) ou a equação (4) é um pouco mais complexo porque a relação entre a tensão cisalhante e o torque no regime elasto-plástico é não linear e requer um procedimento incremental para cálculo de $\tau_{0}$. Para determinar o valor de $\tau_{0}$ é conveniente reescrever a equação (4) na seguinte maneira:

$$
\tau_{\mathrm{o}}=\tau_{\mathrm{i}}\left(\mathrm{r}_{\mathrm{i}} / \mathrm{r}_{\mathrm{o}}\right)^{3}+\left(1 / 2 \pi \mathrm{r}_{\mathrm{o}}{ }^{3}\right)[3 \mathrm{~T}+\phi /(\mathrm{d} \phi / \mathrm{dT})]
$$

pois $\theta=\phi / \mathrm{L}, \theta /(\mathrm{d} \theta / \mathrm{dT})=\phi /(\mathrm{d} \phi / \mathrm{dT})$ e a derivada $\mathrm{d} \phi / \mathrm{dT}$ é calculada a partir da equação (8).

No início da curva $\tau i=\tau o \times(\mathrm{di} / \mathrm{do})$ e $\theta \times \mathrm{dT} / \mathrm{d} \theta \cong T$, então os valores para $\tau$ o são calculados substituindo estas aproximações na equação (1). Para ângulos maiores a parcela não linear é mais significativa e o cálculo segue o procedimento incremental descrito por BROWN [5].

Após a construção da curva $\tau \times \gamma$ os parâmetros do material são obtidos em duas etapas. Primeiro é avaliado o valor de $\mathrm{G}$ com ajuste linear dos dados próximos da origem. Na Figura 11a são mostrados os valores obtidos com a equação (12) e o ajuste linear para $\gamma<0.001$.
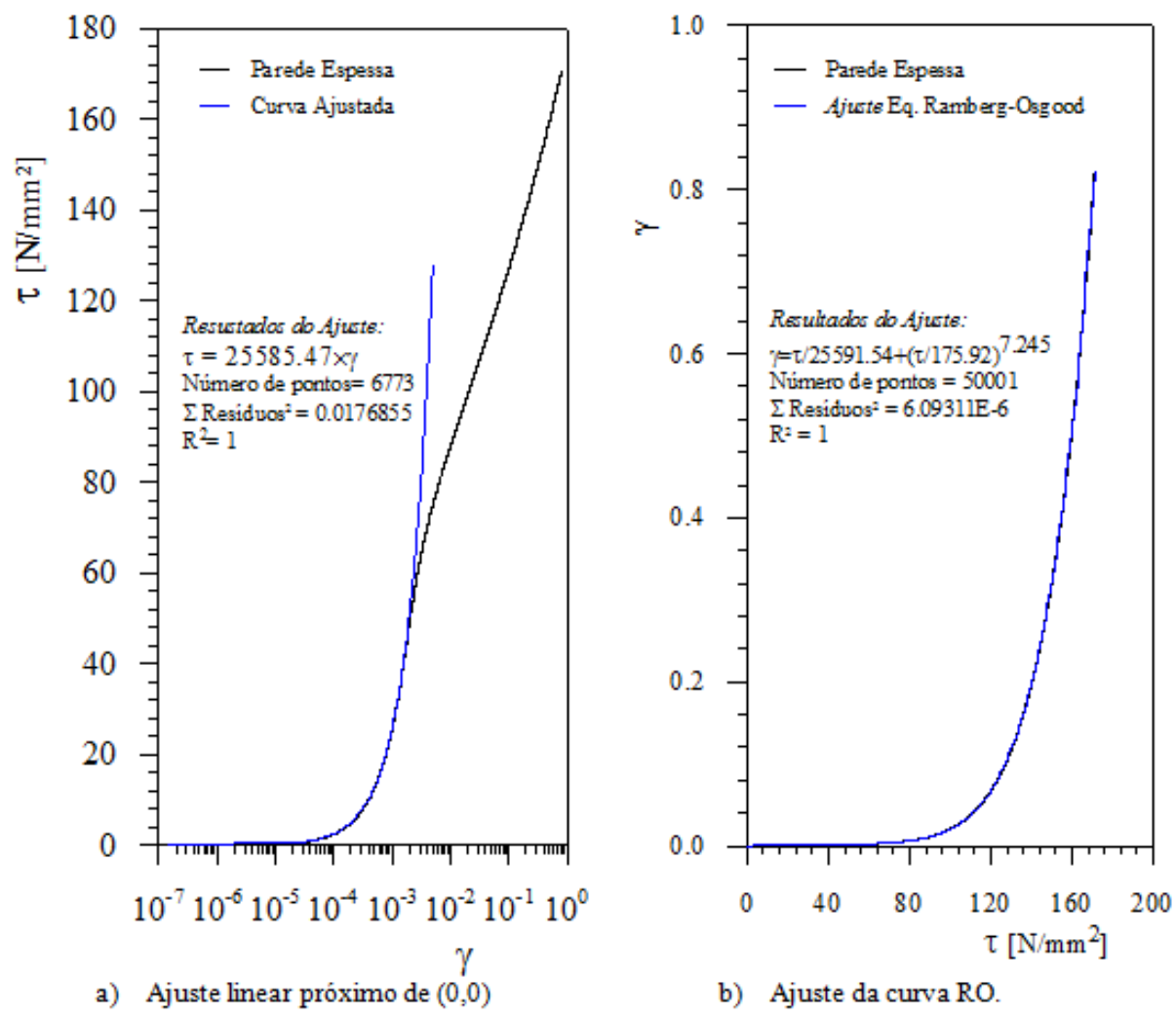

b) Ajuste da curva RO.

Figura 11: Ajustes para o pós-processamento das tensões com a equação (12).

A escala logarítmica na Figura 11a permite visualizar melhor os pontos experimentais e a reta ajustada na região linear para valores pequenos da deformação cisalhante. Após esta etapa os outros dois parâmetros são determinados empregando o Método dos Mínimos Quadrados (MMQ) para minimizar a diferença entre os valores obtidos experimentalmente e os valores calculados com a expressão de RO. A expressão obtida para $\gamma(\tau)$ é apresentada na equação (13) e a curva aproximada pode ser vista na Figura 11b.

$\gamma \cong\left[\left(\tau / 25585,47 \times 10^{6}\right)+\left(\tau / 175,92 \times 10^{6}\right)^{7,245}\right]$ 


\section{CONCLUSÃO}

A principal característica do torcímetro digital descrito neste trabalho é a medição da variação angular sem contato. Bons resultados foram obtidos com um protótipo deste sensor em testes de calibração e ensaios de torção com corpos de prova tubulares. A montagem do dispositivo sobre o corpo de prova, preparação da amostra para o ensaio e ligação do sistema completo de medição são simples comparadas a dispositivos para medição do deslocamento angular encontrados na literatura.

A montagem deste sensor na região central do corpo de prova com diâmetro externo constante permite determinar a variação angular entre dois pontos nesta região e elimina os erros com origem nas deformações angulares das extremidades do corpo de prova e eventuais folgas/deformações do sistema de fixação do corpo de prova na máquina de torção.

A medição do ângulo de torção é realizada sem contato e praticamente não existe limite para o ângulo máximo do ensaio. $\mathrm{O}$ valor lido no encoder digital é corrigido pela relação de transmissão de movimento entre as duas engrenagens, 18/66. Para ângulos menores que $6^{\circ}$ as medições mostraram erros compatíveis com as especificações da placa AS5048A e com valor máximo de $0,04^{\circ}$. Na faixa de $0^{\circ}$ a $450^{\circ}$ verificou-se que a diferença máxima com relação à referência foi de $0,19^{\circ}$.

O custo para confecção deste sistema de medição, em torno de US $\$ 150,00$, pode ser considerado relativamente baixo para este tipo de sensor. Neste valor estão incluídos: matéria prima, usinagem, rolamentos, engrenagens, elementos de fixação, placa AS5048A, microprocessador arduino Mega 2560, cabos e conectores.

Os parâmetros do material obtidos pelo ajuste da equação de Ramberg-Osgood a partir das tensões calculadas pelos métodos indicados para tubos de paredes fina e espessa apresentaram valores similares para $\mathrm{H}$ e n. Os valores obtidos para o parâmetro $\mathrm{G}$ apresentaram uma variação de aproximadamente $11 \%$ entre os dois métodos. O corpo de prova utilizado neste trabalho apresenta dimensões fora da faixa teoricamente indicada como tubo de parede fina, ou seja, relação entre diâmetro médio e espessura do tubo é menor que 10:1. Devido a isto, a consideração que a tensão é distribuída uniformemente ao longo da parede do tubo pode conduzir a erros, dessa forma, o método para tubos de paredes espessas apresentou maior confiabilidade para o corpo de prova utilizado.

\section{AGRADECIMENTOS}

Os autores agradecem aos apoios financeiros fornecidos pelo Conselho Nacional de Desenvolvimento Científico e Tecnológico (CNPq), projeto número 304735/2015-9 da Coordenação de Aperfeiçoamento de Pessoal de Nível Superior (CAPES) - Brasil- Código Financeiro 001" e pela FAPESC (processos 466/2017TR 765/0217 e 937/2019-TR649/2019).

\section{BIBLIOGRAFIA}

[1] SHARIFIMEHR, S., FATEMI, A., "Evaluation of Estimation Methods for Shear Fatigue Properties and Correlations with Uniaxial Fatigue Properties for Steeels and Titanium Alloys", IMATEC Web of Conferences, v. 165, pp. 4-11, 2018.

[2] E 2207: Standard Practice for Strain Controlled Axial-Torsional Fatigue Testing with Thin-Walled Tubular Specimens. ASTM - American Society for Tenting and Materials, 2015.

[3] FACHERIS, G., JANSSENS, K.G.F., FOLETTI, S., "Multiaxial fatigue behavior of AISI 316L subjected to straincontrolled and ratcheting paths", International Journal of Fatigue, v. 68, pp. 195-208, 2014

[4] WU, H.C., XU, Z., WANG, P.T., “Torsion test of Aluminum in the Large Strain Range”, International Journal of Plasticity, v. 13, n. 10, pp. 873-892, 1998.

[5] BROWN, M.W. "Torsional stresses in tubular specimens" Journal of strain analysis, v. 13, n. 1, pp. 6, 1978.

[6] WU, H.C., XU, Z., WANG, P.T.," The Shear Stress-Strain Curve determination from Torsion Test in the Large Strain Range", Journal of testing and evaluation, v. 20, n. 6, pp. 396-402, 1992.

[7] NADAI, A.L. Theory of flow and fracture of solids. Vol.1, New York, McGraw-Hill, 1950.

[8] UNITED STATES PATENT OFFICE. John Hammond Smith. Twist Meter. Serial N 119661, October 9, 1928.

[9] Data Sheet WP500.90 TORSIOMETER, https://www.gunt.de/images/datasheet/1646/WP-500.90-Torsiometer-gunt1646-pdf_1_en-GB.pdf. Acessado em Outubro de 2020.

[10] SANTOS, D.C., BARBIERI, R., "Projeto, construção e teste de um Torcímetro". Matéria (Rio J.), v.16, n.2, pp.703$713,2011$.

[11] User Guide SM 1001, http://cste.sut.ac.th/miscste/company/manual/SM1001.pdf. Acessado em Outubro de 2020. 
[12] SWIFT, W.A.C. “The acquisition and processing of torque-twist data in torsion testing with overstrain", Strain, p. $161-172,1974$.

[13] SCHERER, J.F., BOM, R.P., BARBIERI, R., "Torsional fatigue fibers reinforced epoxy resin composites”. Engineering Research Express, v.2, 2020.

[14] PINTÃO, C.A.F., PIEDADE, L.P., BORALI, E., "System to measure torsion modulus of polymers using the deformation energy method." Polímeros: Ciência e Tecnologia, e2019031. https://doi.org/10.1590/0104- 1428.01019, v. 29, n.3, 2019.

[15] KAKALEY, D.E., ALTIERI, R.E., BUCKNER, G.D., "Non-contacting measurement of torque and axial translation in high-speed rotating shafts", Mechanical Systems and Signal Processing, v. 138, 2020.

[16] LIU, D., PENG, K., HE, Y., "Direct measurement of torsional properties of single fibers", Measurement Science and Technology, v. 27, 2016.

[17] YU, L., LIU, D., PENGAND, K., et al.“An improved torsion pendulum based on image processing for single fibers”, Measurement Science and Technology, v. 27, 2016.

[18] TORO, S.A., ARANDA, P.M., HERRERA, C.M.G., et al. "Analysis of the Elastoplastic Response in the Torsion Test Applied to a Cylindrical Sample”, Materials, v. 12, n. 19, p. 1-16, 2019.

[19] TRAPHÖNER, H., CLAUSMEYER, T., TEKKAYA, A.E., "Methods for measuring large shear strains in in-plane torsion tests", Journal of Materials Processing Tech., Disponível em: https://doi.org/10.1016/j.jmatprotec.2019.116516, 2019.

[20] MIR, A., HASSAN, A., "Low cost solution of performing torsion testing of materials", In: International Conference on Mechatronics and Automation, Takamatsu, Japan, 06-09 August 2017.

[21] AS5048A HIGH-RESOLUTION POSITION SENSOR, https://ams.com/as5048a. Acessado em Dezembro de 2018.

\section{ORCID}

Maurício Ireno Betemps

Renato Barbieri

Guilherme Ourique Verran https://orcid.org/0000-0001-9626-5190

https://orcid.org/0000-0001-7180-1014

https://orcid.org/0000-0001-7814-1493 